Supporting Information

\title{
Interplay of Fermi Level Pinning, Marcus Inverted Transport, and Orbital Gating in Molecular Tunneling Junctions
}

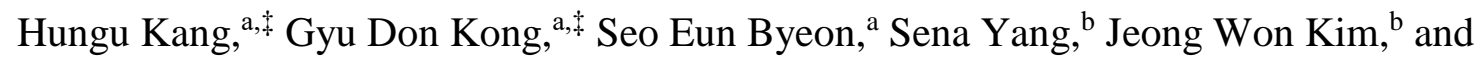
Hyo Jae Yoon $^{\text {a* }}$

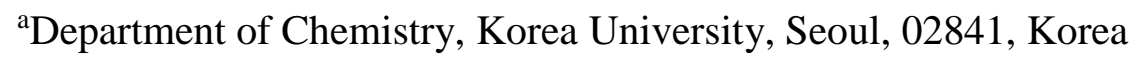

${ }^{\mathrm{b}}$ Korea Research Institute of Standards and Science (KRISS), Daejeon, 34113, Korea

\$These authors contributed to this work equally. 


\section{$\underline{\text { Table of Contents }}$}

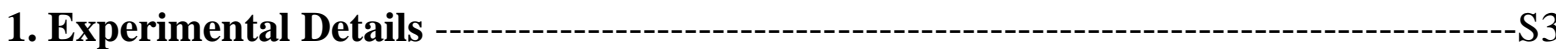

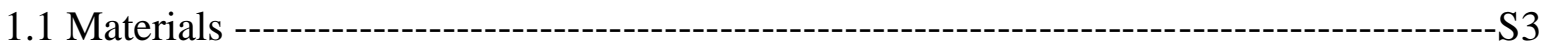

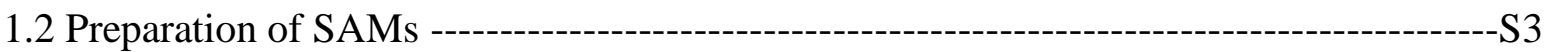

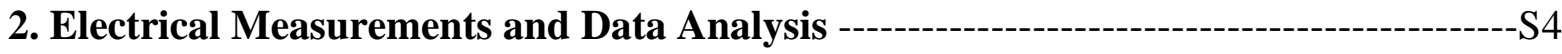

2.1 Preparation of Tethered EGaIn Junction -------------------------------------------------S4

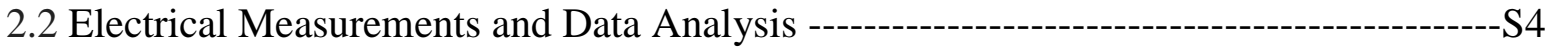

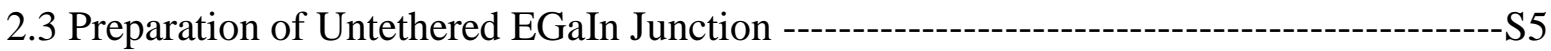

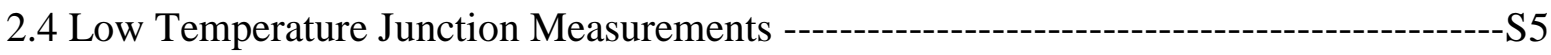

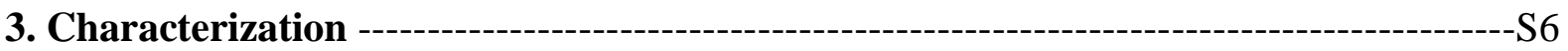

3.1 X-ray Photoelectron Spectroscopy (XPS) Measurements ----------------------------------S6

3.2 Ultraviolet Photoelectron Spectroscopy (UPS) Measurements ----------------------------S6

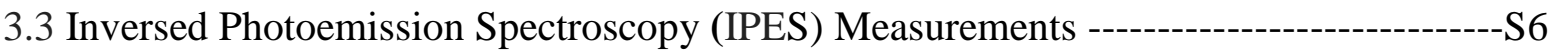

3.4 Near Edge X-ray Adsorption Fine Structure (NEXAFS) Measurements -----------------S7

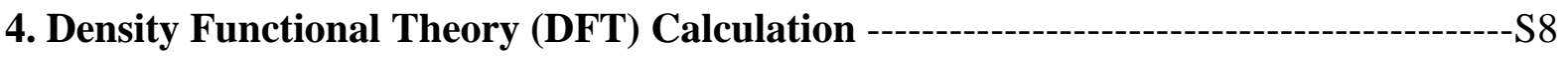

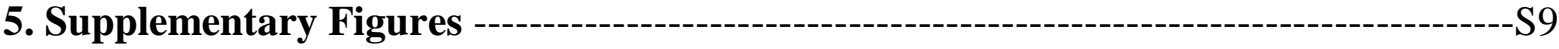

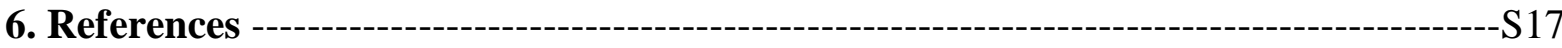




\section{Experimental Details}

\subsection{Materials}

All reagents were used as supplied unless otherwise specified. All organic solvents were purchased from Sigma-Aldrich and Daejung. High purity eutectic gallium-indium (EGaIn; 99.99\%) was obtained from Sigma-Aldrich and used as supplied. 2,2'-Bipyridylterminated $n$-undecanethiol ( $\mathrm{HSC}_{11} \mathrm{BIPY}$ ) was synthesized following the procedures reported in the literature. ${ }^{1}$ All thiol derivatives were stored under $\mathrm{N}_{2}$ atmosphere and $<4{ }^{\circ} \mathrm{C}$. Gold thin films ( 300 nm) were deposited onto silicon wafer (100 $\mathrm{mm}$ in diameter; $1-10$ ohm-cm, $525 \pm 50$ microns thick) by e-beam evaporator (ULVAC). For template-stripping, photo-curable polymer was purchased from Norland (NOA81) and used as supplied.

\subsection{Preparation of SAMs}

We prepared SAMs following the procedure reported previously. ${ }^{2}$ Briefly, toluene (anhydrous, 99.9\%) solution (total concentration: $3 \mathrm{mM}$ ) containing $\mathrm{HSC}_{11} \mathrm{BIPY}$ was added to a vial. The solution was sealed and degassed by bubbling $\mathrm{N}_{2}$ through the solution for $c a$. 10 min. Template-stripped metal substrates were prepared following the procedure reported previously. ${ }^{3}$ A freshly prepared $\mathrm{Au}^{\mathrm{TS}}$ chip was rinsed with pure toluene, and placed to the solution with the exposed metal face up. The vial was then filled with $\mathrm{N}_{2}$. After $3 \mathrm{~h}$ incubation at room temperature, the SAM-bound $\mathrm{Au}^{\mathrm{TS}}$ chip was removed from the solution and rinsed by repeatedly dipping the chip into clean toluene $(3 \times 1 \mathrm{~mL})$. The solvent on the SAM was then evaporated in air for a few seconds. 


\section{Electrical Measurements and Data Analysis}

\subsection{Preparation of Tethered EGaIn Conical Tip}

A conical tip of EGaIn was formed following the method reported in the literature. ${ }^{4}$ Briefly, a $10 \mu \mathrm{L}$ gas-tight syringe was filled with EGaIn ( $\geq 99.99 \%$, Aldrich). A drop of EGaIn was pushed to the tip of the syringe needle, the hanging drop was brought into contact with a surface on which the EGaIn could stick (e.g., an oxidized Ag surface), and the needle gently pulled away from the drop using a micromanipulator. Upon breaking from the bulk EGaIn on the surface, a conical tip was obtained. A conical tip of EGaIn was newly formed every junction in order to eliminate complexities that may arise from contamination of the EGaIn surface by volatile organics in air. In cases that visible whiskers formed during tip fabrication, the tip was discarded, and a new tip was formed.

\subsection{Electrical Measurements and Data Analysis}

Junction formation and measurements were done following the method reported in the literature. ${ }^{1}$ Briefly, a SAM was gently brought into contact first with a gold metal electrode. Then using a micromanipulator, a conical tip of EGaIn was gently brought into contact with the surface of SAM. The SAM-bound bottom electrode was grounded, and the EGaIn top electrode was biased.

The diameter of the contact area was measured at high magnification. Assuming a circular contact, the area was derived from the measured diameter from which the current densities $\left(J, \mathrm{~A} / \mathrm{cm}^{2}\right)$ were calculated. The contact and presence of a SAM was confirmed by running a single $J$ - $V$ scan after which 20 more scans were run if there was indication of contact and tunneling. A trace is based on the following voltage sweep sequence: $0 \mathrm{~V} \rightarrow$ $+1.0 \mathrm{~V} \rightarrow 0 \mathrm{~V} \rightarrow-1.0 \mathrm{~V} \rightarrow 0 \mathrm{~V}$. Therefore, one trace corresponds to two scans. The total 
number of working junctions versus those that shorted was used to calculate the yield (\%) of working junctions. Shorts have been usually excluded prior to analysis. Shorts clearly do not give information about the SAM and can bias distributions of current density toward high values. ${ }^{5}$ Thus, when one performs operations on the raw distribution of logcurrent density $(\log |J|)$, one discards values corresponding to shorts. Shorts are defined as values of current that reach the compliance limit of our electrometer $( \pm 0.105 \mathrm{~A})$. For generating histograms, we kept the bin-size of $r$ histograms constant, making the resolution of mean values (the width of each bean is $\sim 0.14$ in a log-scale).

\subsection{Preparation of Untethered EGaIn Junction}

For low temperature experiments, EGaIn conical tip was untethered following the previously reported method. ${ }^{4}$ Tethered EGaIn conical tip was first prepared and brought to a surface of SAM for top-contact. A few microgram of photo-curable polymer was placed near the formed junction. Immediately the polymer overspread the junction, which could be confirmed by optical microscopy, we flooded it with ultraviolet (UV) light using a handheld lamp for few seconds. For untethering, the syringe was lifted up using a micromanipulator.

\subsection{Low Temperature Junction Measurements}

The temperature dependent measurements were performed with a cryogenic probe station in vacuum $\left(1 \times 10^{-3}\right.$ bar $)$. The devices were cooled with liquid nitrogen from 298 to $118 \mathrm{~K}$ over the course of two hours. At intervals of $10 \mathrm{~K}$, we contacted the $\mathrm{Au}^{\mathrm{TS}}$ and $\mathrm{Ga}_{2} \mathrm{O}_{3} / \mathrm{EGaIn}$ electrodes with the probes and recorded three $J(V)$ curves while keeping the temperature constant. For measurements, the $\mathrm{Ga}_{2} \mathrm{O}_{3} / \mathrm{EGaIn}$ top-electrode was biased and the $\mathrm{Au}^{\mathrm{TS}}$ bottom-electrode was grounded. 


\section{Characterization}

\subsection{X-ray Photoelectron Spectroscopy (XPS) Measurements}

The XPS measurements were carried out on a Thetaprobe (Thermo) with a monochromated $\mathrm{Al} \mathrm{Ka}(1486.6 \mathrm{eV})$ source. The measurements were done at room temperature in a vacuum of $6.7 \times 10^{-8} \mathrm{~Pa}$. The binding energies were calibrated by setting $\mathrm{Au} 4 \mathrm{f}_{7 / 2}$ of bulk metals to values $84.0 \mathrm{eV} .{ }^{6}$ The XPS N1s and S2p lines were detected to study the $\mathrm{SC}_{11} \mathrm{BIPY} \mathrm{SAM}$ and consistent with the literature results. ${ }^{1-3}$ The peak shapes of the core level photoelectron spectra were analyzed with a XPS Peak Fit program. A Shirley-type background correction was utilized. The $S 2 \mathrm{p}_{3 / 2}$ and $S 2 \mathrm{p}_{1 / 2}$ lines were fitted with a fixed binding energy difference of $1.18 \mathrm{eV}$ and an intensity ratio of 2:1, reflecting the multiplicity of the $S 2 p_{3 / 2}$ and $S 2 p_{1 / 2}$ energy levels. ${ }^{7}$ The reproducibility of the data was confirmed by measuring the data in different samples.

\subsection{Ultraviolet Photoelectron Spectroscopy (UPS) Measurements}

The UPS measurements were carried out on Thetaprobe (Thermo) to determine the Fermi level and the HOMO level of $\mathrm{Au}^{\mathrm{TS}} / \mathrm{SC}_{11} \mathrm{BIPY}$ SAM. The HOMO level was determined by the following equation, HOMO level $=h v-\left(E_{\text {cutoff }}-E_{\text {onset }}\right),{ }^{8}$ where $h v$ is incident photon energy $(21.2 \mathrm{eV})$ of He I source, the high binding energy cutoff ( $\left.E_{\text {cutoff }}\right)$ and HOMO region $\left(E_{\text {onset }}\right)$ are the turning points.

\subsection{Inversed Photoemission Spectroscopy (IPES) Measurements}

In the IPES measurement, isochromatic mode with scanning electron energy was employed. The electron source (PSP Vacuum Technology) with well-defined energy emits low energy electrons on the sample and emitted photons are detected by the $\mathrm{BaF}_{2}$ 
window/KBr-coated micro-channel plate (Photonics). The energy scales of IPES spectra is aligned by matching the Fermi energies $\left(E_{\mathrm{F}}\right)$ of a clean $\mathrm{Au}(111)$ single crystal. The total instrumental energy resolution was less than $0.1 \mathrm{eV}$ for IPES measurements, respectively. ${ }^{9}$

\subsection{Near Edge X-ray Adsorption Fine Structure (NEXAFS) Measurements}

The NEXAFS measurements were performed at 4D PES beam line of Pohang Accelerator Laboratory (PAL) in South Korea. We used the partial electron yield (PEY) detection mode for the NEXAFS spectra by recording the sample current normalized to a signal current, which was measured simultaneously using a gold mesh in ultrahigh vacuum $\left(<10^{-9}\right.$ Torr $)$. In this case, a $p$-polarized $(\sim 85 \%)$ synchrotron photon beam had an energy in the range of 270-325 eV and spectral energy resolution of $\Delta E=150 \mathrm{meV}$. Carbon K-edge angle-dependent NEXAFS from $30^{\circ}$ to $70^{\circ}$ is sensitive to the $\pi^{*}$ antibonding orbital along the $\pi$ bond (Fig. S5). The NEXAFS spectra of typical $\pi$-conjugated organic semiconductors shows a $\mathrm{C} 1 \mathrm{~s}$ to $\pi^{*}$ resonance at $284-287 \mathrm{eV}$. The average molecular orientation was determined by analyzing the change in the intensity of the $\pi^{*}(\mathrm{C}=\mathrm{C})$ and $\sigma^{*}(\mathrm{C}-\mathrm{C})$ resonance value at 284.5 and $293.0 \mathrm{eV}$, respectively, as the angle of incidence of the X-ray beam was changed. From the NEXAFS results, LUMO energy level ( $\varepsilon$ LUMO) was obtained following the method reported in the literature. ${ }^{10}$ Based on the first resonance peak $\left(4 \mathrm{e}_{1 \mathrm{~g}}\right)$ of the Carbon K edge spectra of NEXAFS, $\varepsilon_{\text {LUMO }}$ was calculated from the difference between photon energy of the $4 \mathrm{e} 1 \mathrm{~g}$ peak $(\mathrm{PE})$ and the $\mathrm{C} 1 \mathrm{~s}$ binding energy $\left(\mathrm{BE}_{\mathrm{C}}\right)$, and the exciton binding energy $\left(\mathrm{BE}_{\mathrm{E}}\right)$ corrected with core-hole using the following Eq. 1:

$$
\varepsilon_{\mathrm{LUMO}}=\mathrm{PE}-\mathrm{BE}_{\mathrm{C}}+\mathrm{BE}_{\mathrm{E}}
$$

The $\mathrm{BE}_{\mathrm{E}}$ value was $500 \mathrm{meV}$, which is a commonly used value for a thin organic film. 


\section{Density Functional Theory (DFT) Calculation}

The DFT calculations were performed to study the molecular electronic characteristics with Gaussian 09 program employing Becke three-parameter hybrid functional and the correlation functional of Lee-Yang-Parr (B3LYP) with a 6-31G(d) basis set. ${ }^{11}$ The density of states (DOS) curve was plotted by using GaussSum3.0 with a broadening of 0.55 $\mathrm{eV} \cdot{ }^{12}$ 


\section{Supplementary Figures and Tables}

Figure S1. Histograms of $\log |J(V)|$ and $\log \left|r^{+}\right|$values for $\mathrm{SC}_{11} \mathrm{BIPY}$ on $\mathrm{Au}^{\mathrm{TS}}$.
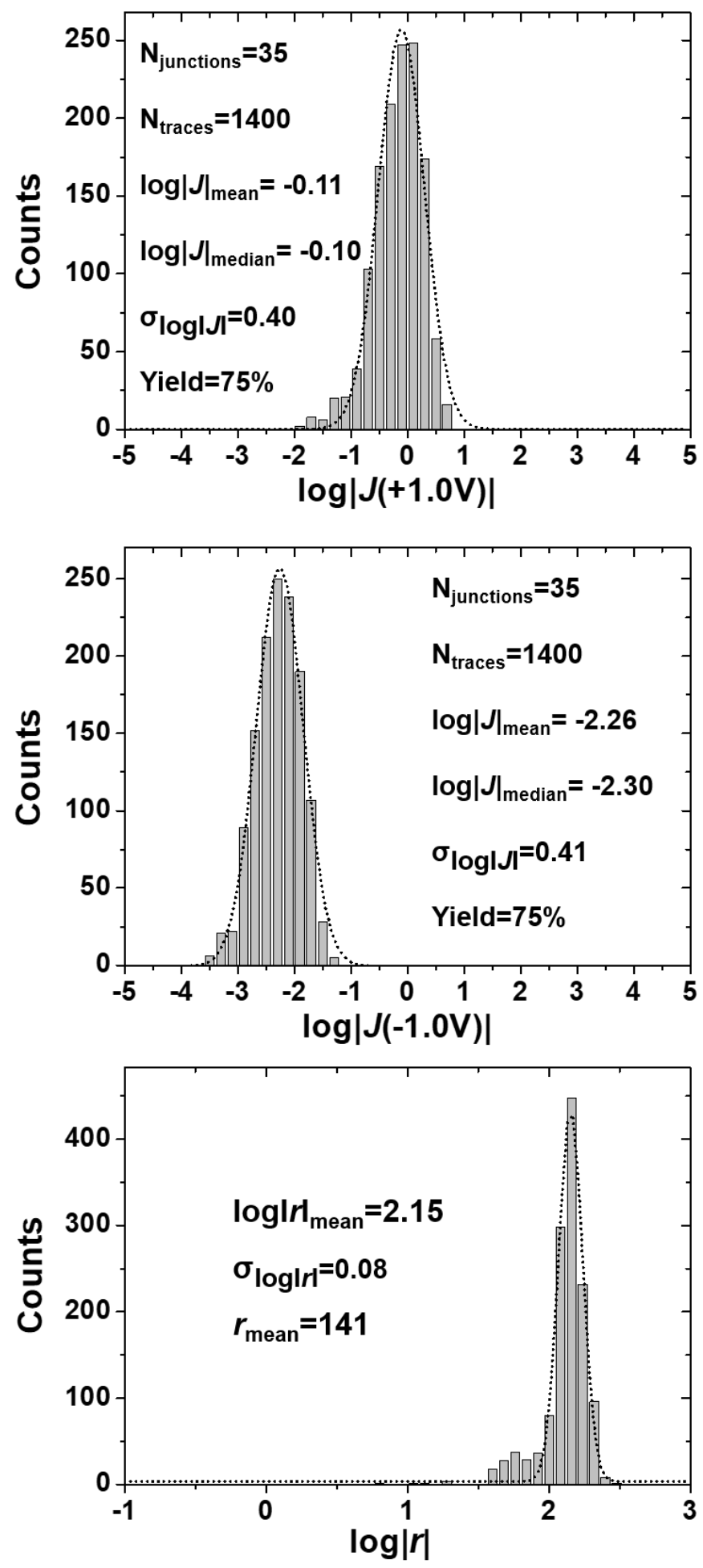
Figure S2. Survey spectra obtained using XPS for the $\mathrm{Au}^{\mathrm{TS}} / \mathrm{SC}_{11} \mathrm{BIPY}$ SAM.

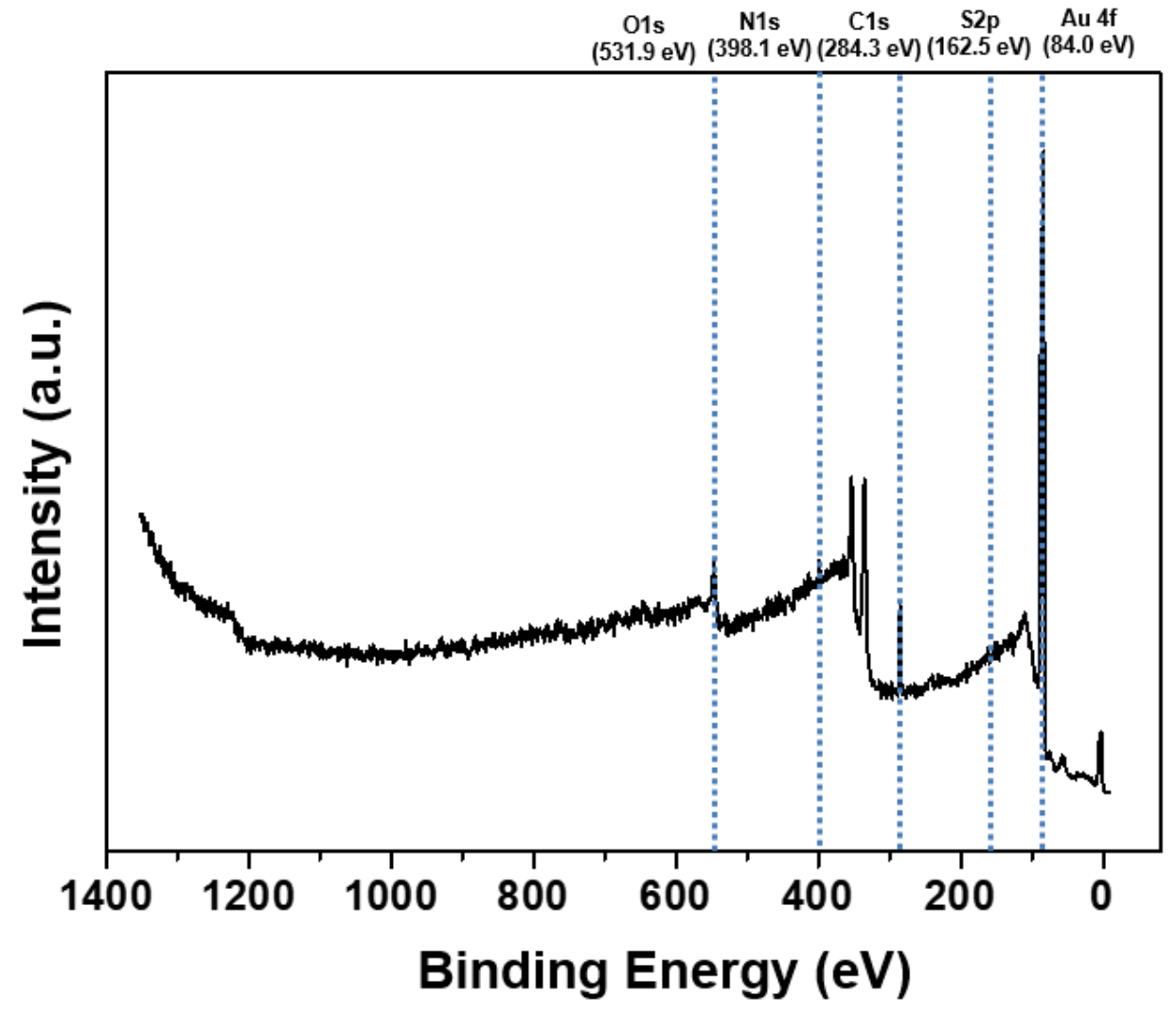


Figure S3. The high resolution XPS spectra of S2p and N1s for pure $\mathrm{SC}_{11} \mathrm{BIPY}$ on $\mathrm{Au}^{\mathrm{TS}}$.
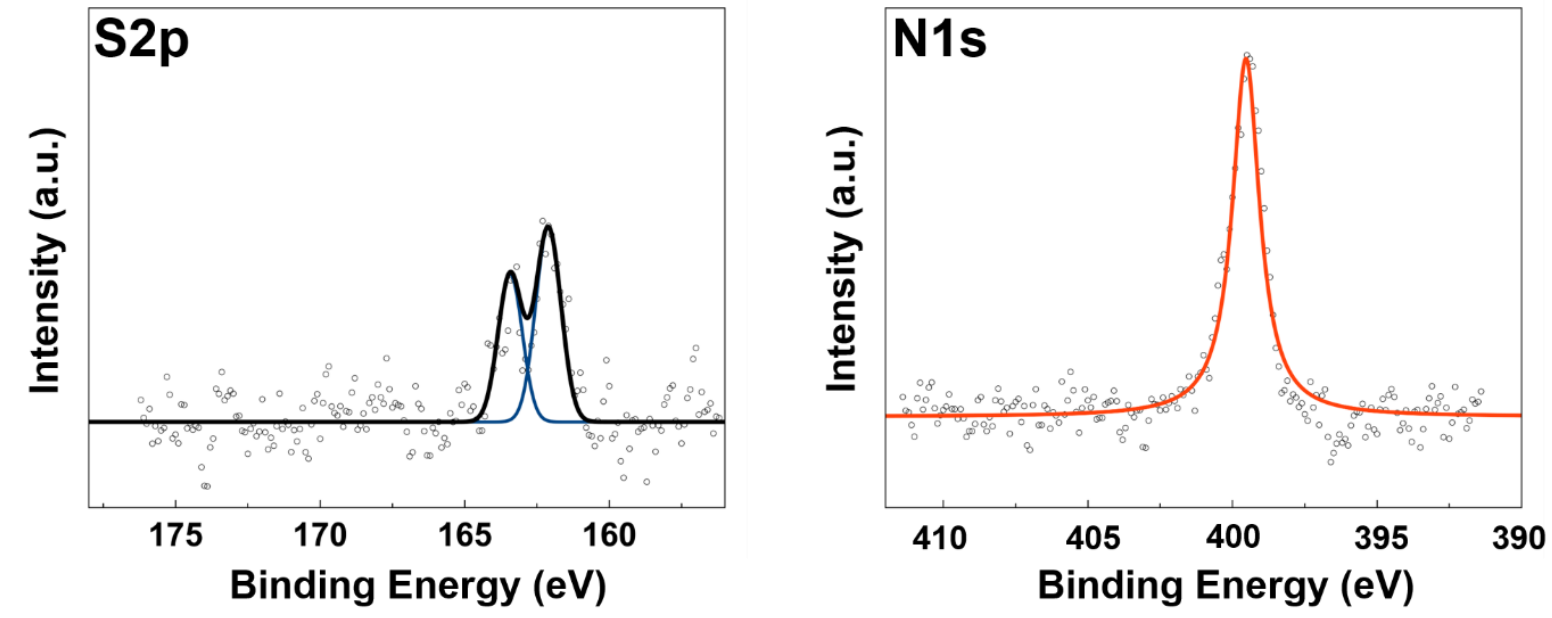
Figure S4. Energy diagrams for Marcus normal charge transfer processes with respect to the reaction coordination $(X) . \lambda$ and $E_{\mathrm{a}}$ are the reorganization energy and the activation energy, respectively.

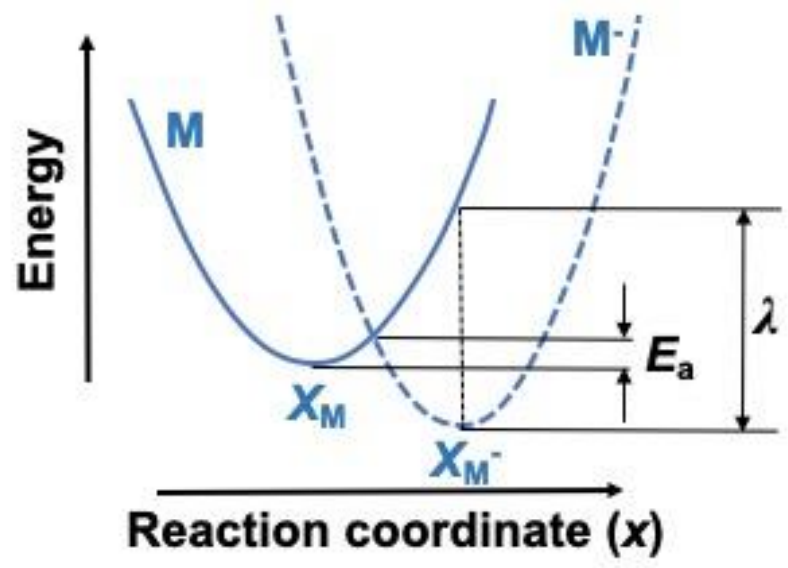


Figure S5. The UPS spectra of bare $\mathrm{Ga}_{2} \mathrm{O}_{3} / \mathrm{EGaIn}$ (black line) and BIPY adsorbed $\mathrm{Ga}_{2} \mathrm{O}_{3} / \mathrm{EGaIn}$ (red line) surfaces.

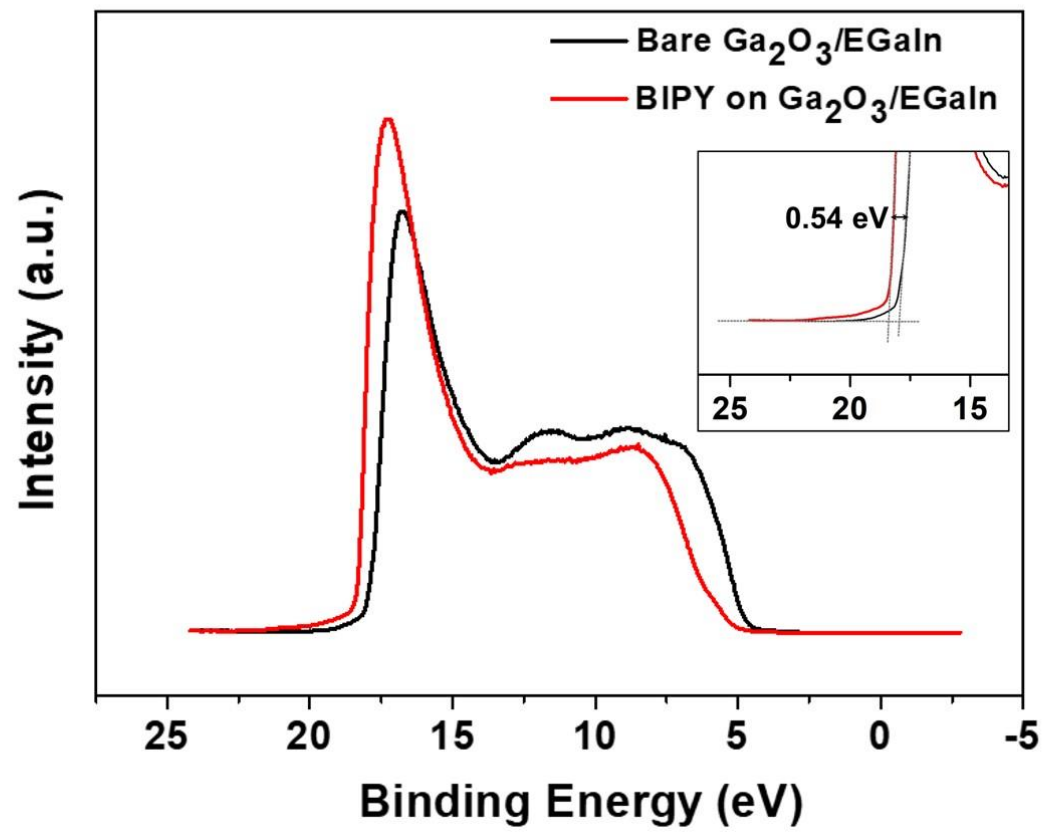


Figure S6. The $\mathrm{C} 1 \mathrm{~s}$ NEXAFS spectra for $\mathrm{SC}_{11} \mathrm{BIPY} \mathrm{SAM}$ on $\mathrm{Au}^{\mathrm{TS}}$ at the grazing X-ray incidence angle of $45^{\circ}$.

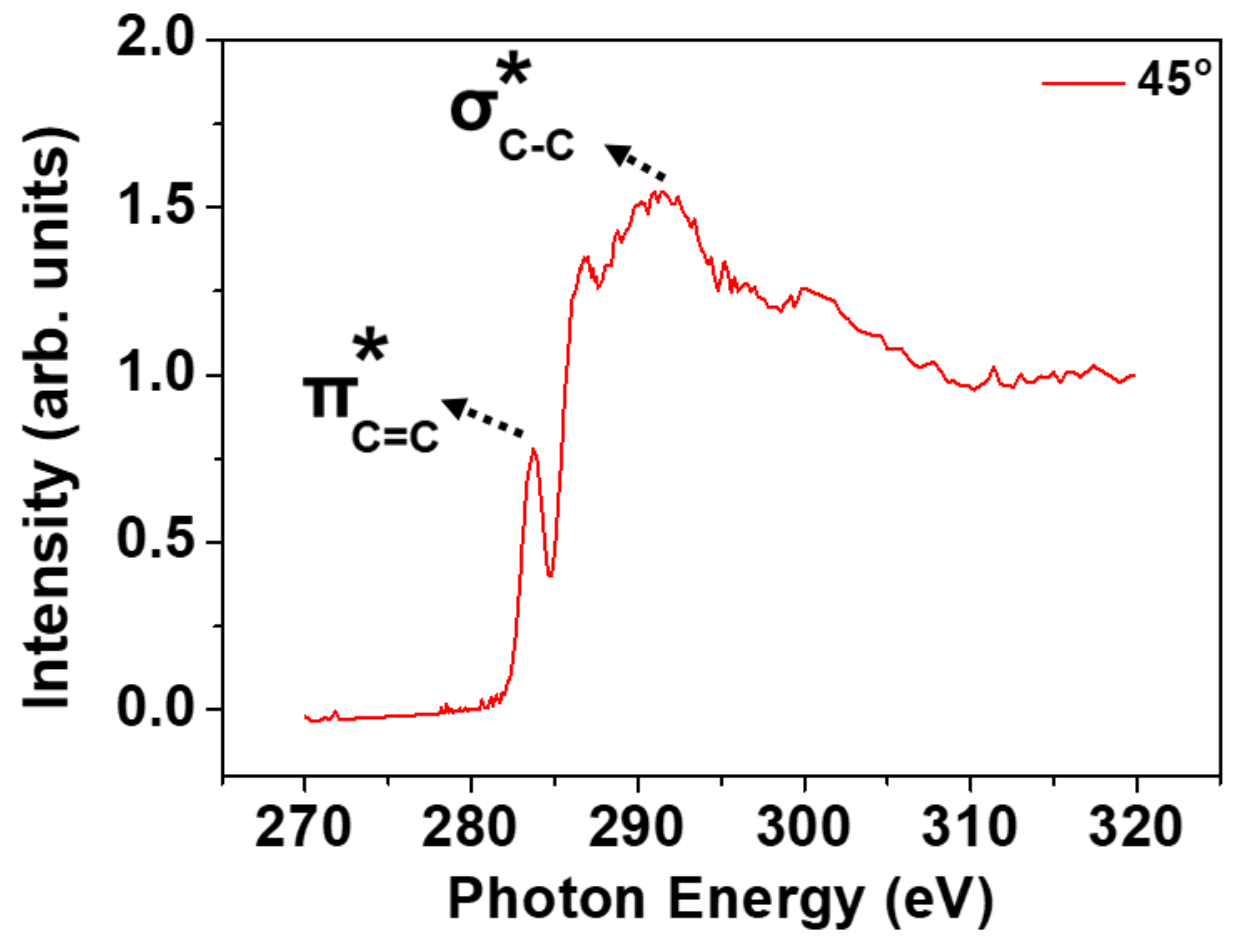


Figure S7. The DFT calculation of model compound ( $\mathrm{HSC}_{11} \mathrm{BIPY}$ in a gas phase).

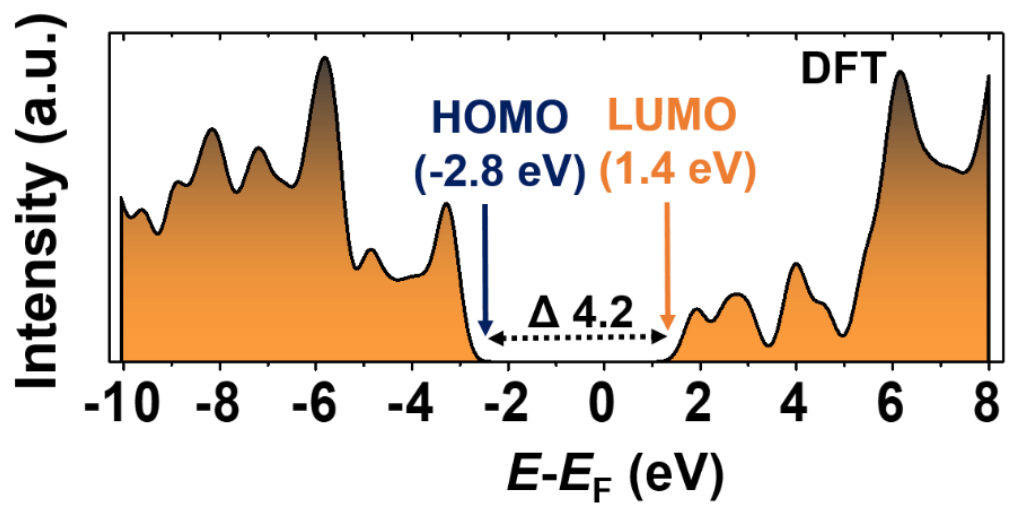


Figure S8. Histograms of $\log |J(V)|$ and $\log |r|$ values for $\mathrm{SC}_{11} \mathrm{BIPY}$ SAMs formed on different bottom electrodes: (a) $\mathrm{Ag}^{\mathrm{TS}}$, (b) $\mathrm{Pt}^{\mathrm{TS}}$ and (c) $\mathrm{Au}^{\mathrm{AD}}$. Data in (a) is taken from reference 2.

(a)
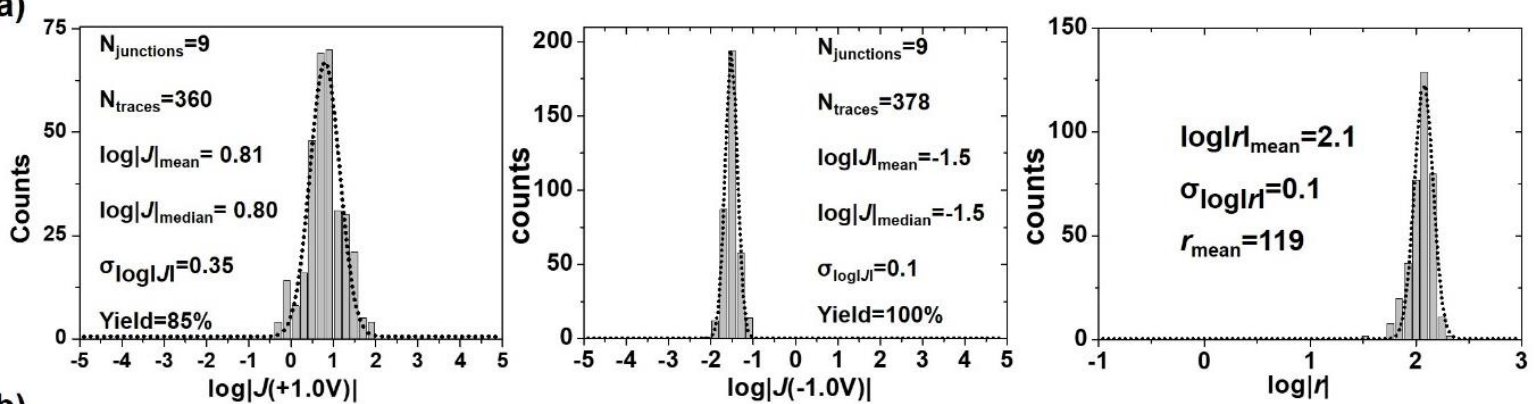

(b)
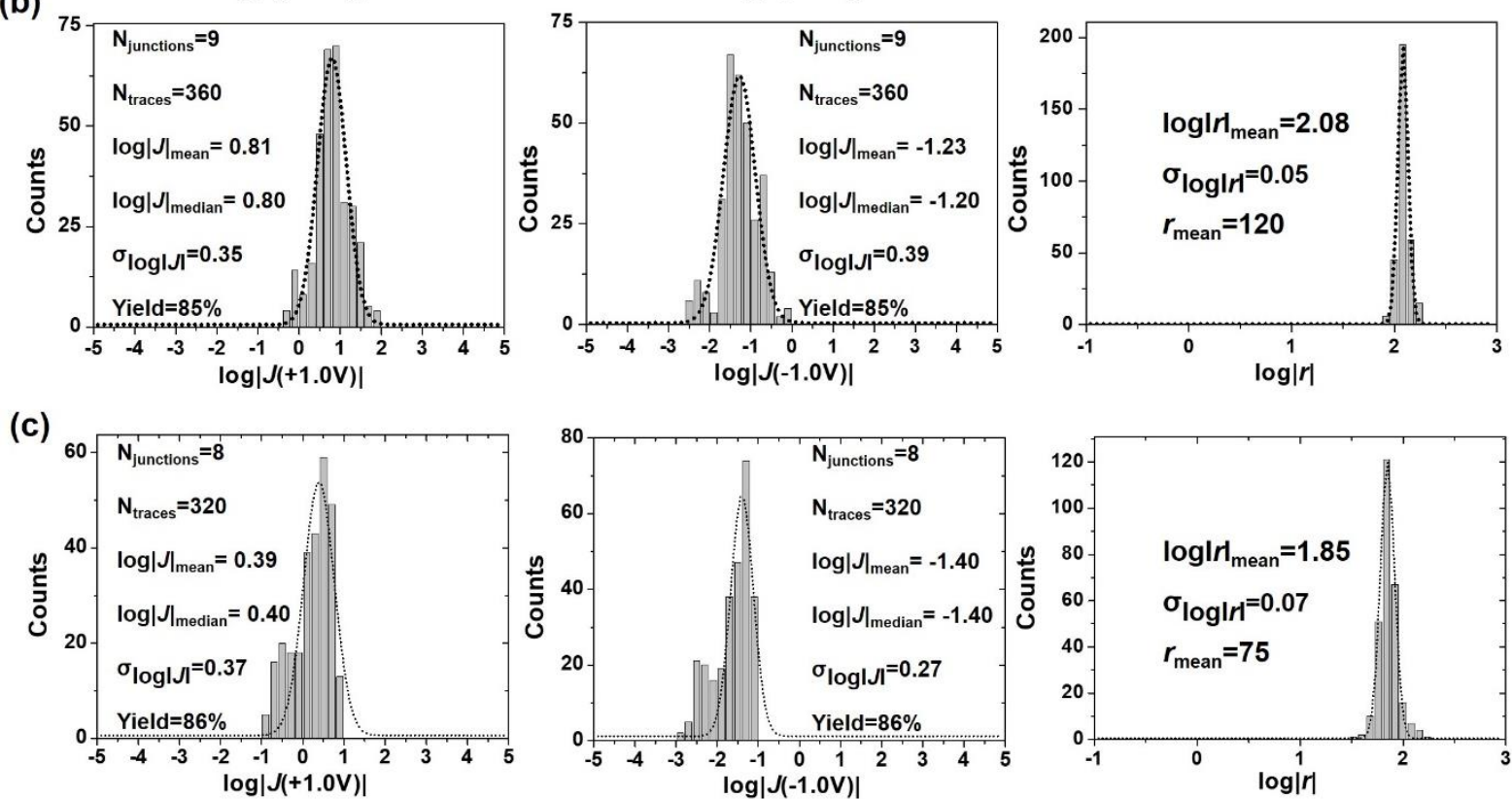


\section{References}

1. Yoon, H. J.; Liao, K.-C.; Lockett, M. R.; Kwok, S. W.; Baghbanzadeh, M.; Whitesides, G. M. Rectification in Tunneling Junctions: 2,2'-Bipyridyl-terminated $n$ Alkanethiolates. J. Am. Chem. Soc. 2014, 136, 17155-17162.

2. Kong, G. D.; Kim, M.; Cho, S. J.; Yoon, H. J. Gradients of Rectification: Tuning Molecular Electronic Devices by the Controlled Use of Different-sized Diluents in Heterogeneous Self-assembled Monolayers. Angew. Chem. Int. Ed. 2016, 55, 10307-10311. 3. Kong, G. D.; Jin, J.; Thuo, M.; Song, H.; Joung, J. F.; Park, S.; Yoon, H. J. Elucidating the Role of Molecule-electrode Interfacial Defects in Charge Tunneling Characteristics of Large-area Junctions. J. Am. Chem. Soc. 2018, 140, 12303-12307. 4. Byeon, S. E.; Kim, M.; Yoon, H. J. Maskless Arbitrary Writing of Molecular Tunnel Junctions. ACS Appl. Mater. Interfaces 2017, 9, 40556-40563.

5. Chiechi, R. C.; Weiss, E. A.; Dickey, M. D.; Whitesides, G. M. Eutectic Galliumindium (EGaIn): A Moldable Liquid Metal for Electrical Characterization of Self-assembled Monolayers. Angew. Chem. Int. Ed. 2008, 47, 142-144.

6. Ishida, T. C., N.; Mizutani, W.; Tokumoto, H.; Kojima, I.; Azehara, H.; Hokari, H.; Akiba, U.; and Fujihira, M. High-Resolution X-ray Photoelectron Spectra of Organosulfur Monolayers on $\mathrm{Au}(111)$ : S(2p) Spectral Dependence on Molecular Species. Langmuir 1999, 15, 6799-6806.

7. Kong, G. D.; Yoon, H. J. Influence of Air-Oxidation on Rectification in Thiol-Based Molecular Monolayers. J. Electrochem. Soc. 2016, 163, G115-G121.

8. $\quad$ Cho, S. J.; Kong, G. D.; Park, S.; Park, J.; Byeon, S. E.; Kim, T.; Yoon, H. J. Molecularly Controlled Stark Effect Induces Significant Rectification in PolycyclicAromatic-Hydrocarbon-Terminated n-Alkanethiolates. Nano Lett. 2019, 19, 545-553.

9. Lim, H.; Kwon, H.; Kim, S. K.; Kim, J. W. Delayed Triplet-State Formation through Hybrid Charge Transfer Exciton at Copper Phthalocyanine/GaAs Heterojunction. J. Phys. Chem. Lett. 2017, 8, 4763-4768.

10. Chen, X.; Roemer, M.; Yuan, L.; Du, W.; Thompson, D.; Del Barco, E.; Nijhuis, C. A. Molecular Diodes with Rectification Ratios Exceeding $10^{5}$ Driven by Electrostatic Interactions. Nat. Nanotechnol. 2017, 12, 797-803.

11. Lee, H.; Lee, J.; Park, S.; Yi, Y.; Cho, S. W.; Kim, J. W.; Kang, S. J. Hole Injection Enhancement of a Single-walled Carbon Nanotube Anode using an Organic Chargegeneration Layer. Carbon 2014, 71, 268-275.

12. O'Boyle, N. M.; Tenderholt, A. L.; Langner, K. M. Cclib: A Library for Packageindependent Computational Chemistry Algorithms. J. Comput. Chem. 2008, 29, 839-845. 